\title{
Piperlongumine promotes autophagy via inhibition of Akt/mTOR signalling and mediates cancer cell death
}

\author{
P Makhov ${ }^{\star}, 1$, K Golovine $^{1}$, E Teper ${ }^{1}$, A Kutikov ${ }^{1}$, R Mehrazin $^{1}$, A Corcoran ${ }^{1}$, A Tulin ${ }^{1}$, R G Uzzo ${ }^{1}$ \\ and V M Kolenko ${ }^{1}$ \\ ${ }^{1}$ Department of Surgical Oncology, Fox Chase Cancer Center of Temple University School of Medicine, Philadelphia, \\ PA 19111, USA
}

Background: The Akt/mammalian target of rapamycin (mTOR) signalling pathway serves as a critical regulator of cellular growth, proliferation and survival. Akt aberrant activation has been implicated in carcinogenesis and anticancer therapy resistance. Piperlongumine (PL), a natural alkaloid present in the fruit of the Long pepper, is known to exhibit notable anticancer effects. Here we investigate the impact of $\mathrm{PL}$ on Akt/mTOR signalling.

Methods: We examined Akt/mTOR signalling in cancer cells of various origins including prostate, kidney and breast after PL treatment. Furthermore, cell viability after concomitant treatment with PL and the autophagy inhibitor, Chloroquine (CQ) was assessed. We then examined the efficacy of in vivo combination treatment using a mouse xenograft tumour model.

Results: We demonstrate for the first time that PL effectively inhibits phosphorylation of Akt target proteins in all tested cells. Furthermore, the downregulation of Akt downstream signalling resulted in decrease of mTORC1 activity and autophagy stimulation. Using the autophagy inhibitor, CQ, the level of PL-induced cellular death was significantly increased. Moreover, concomitant treatment with $\mathrm{PL}$ and $\mathrm{CQ}$ demonstrated notable antitumour effect in a xenograft mouse model.

Conclusions: Our data provide novel therapeutic opportunities to mediate cancer cellular death using PL. As such, PL may afford a novel paradigm for both prevention and treatment of malignancy.

Naturally-occurring substances are the most dependable resource for therapeutic development. A variety of FDA approved antineoplastic agents, including docetaxel and topotecan, have demonstrated clinical utility based on ongoing investigations of natural products. Piperlongumine (PL), a natural alkaloid of the Long pepper (Piper longum), exhibits numerous key biological activities. In addition to its insecticidal and bactericidal capabilities (Yang et al, 2002), recent literature points to the fact that PL is capable of hindering the growth of sarcoma, bladder, breast, melanoma and lung tumours in vitro and in vivo (Bezerra et al, 2008; Raj et al, 2011). The ability of PL to target the cellular stress response through direct inhibition of Glutathione S-transferase pi 1 (GSTP1) subsequently inducing of intracellular reactive oxygen species (ROS) accumulation promoting selective killing of cancer cells was initially demonstrated by Raj et al (2011). Furthermore, PL has minimal high-dose acute toxicity, and does not appear to significantly affect any biochemical, haematologic and histopathologic parameters in animal models (Raj et al, 2011). In addition, the ability of PL to downregulate NF- $k$ B activation was demonstrated by our and other studies (Han et al, 2013). Moreover, PL induces rapid depletion of the androgen receptor (Golovine et al, 2013) in prostate cancer cells.

The serine/threonine kinase Akt functions as a critical control point of multiple upstream signalling pathways involving receptor tyrosine kinase stimulation. Activation of Akt promotes cellular growth and proliferation as well as inhibition of apoptosis 
(Majewski et al, 2004). Akt aberrant activation has been implicated in the development of malignancy and anticancer therapy resistance (Gutierrez et al, 2011; Opel et al, 2011).

An effector of Akt functioning is the mammalian target of rapamycin (mTOR), a serine/threonine kinase. A number of recent publications dealing with mechanistic actions of mTORC1 complex provide clear evidence that in addition to its wellestablished effects it also acts as a key checkpoint in signalling pathways regulating autophagy (Alers et al, 2011). Autophagy has been characterised as critical homeostatic and adaptive process that serves to deliver cytoplasmic proteins and organelles to the lysosomes for digestion.

The overall clinical picture, however, is not well defined. Studies of autophagy in different cell types under a spectrum of various cellular conditions provide conflicting evidence regarding its role in cellular death (Debnath et al, 2005; Levine and Kroemer, 2008; White and DiPaola, 2009). Whereas the inhibition of autophagy appears to enhance the sensitivity of tumour cells towards antineoplastic drugs (Amaravadi et al, 2007), the induction of autophagy may promote tumour cell death in those with heightened resistance to apoptosis.
In our present study, we provide first evidence that PL is capable of stimulating autophagy in various cancer cell lines, including prostate, kidney and breast. It does so via ROS-dependent direct downregulation of Akt/mTOR signalling, subsequently promoting increased autophagy in the aforementioned cells. Additionally using autophagy inhibitor, Chloroquine (CQ), we were able to modulate PL-induced cellular death. Furthermore, CQ significantly increased antitumor activity of PL in the xenograft mouse model. Taken together, our data provide novel pathways to potentially mediate cancer cell death by PL-induced autophagy.

\section{MATERIALS AND METHODS}

Cells and culture conditions. Androgen-independent human PC-3 prostate cancer cells, breast cancer MCF-7 cells, renal carcinoma 786-O cells and HEK-293T kidney cells were obtained from ATCC (Rockville, MD, USA). Cells were cultured in RPMI 1640 (BioWhittaker, Walkersville, MD, USA) supplemented with 10\% FBS (Hyclone, Logan, UT, USA), penicillin $\left(100 \mathrm{U} \mathrm{ml}^{-1}\right)$, streptomycin

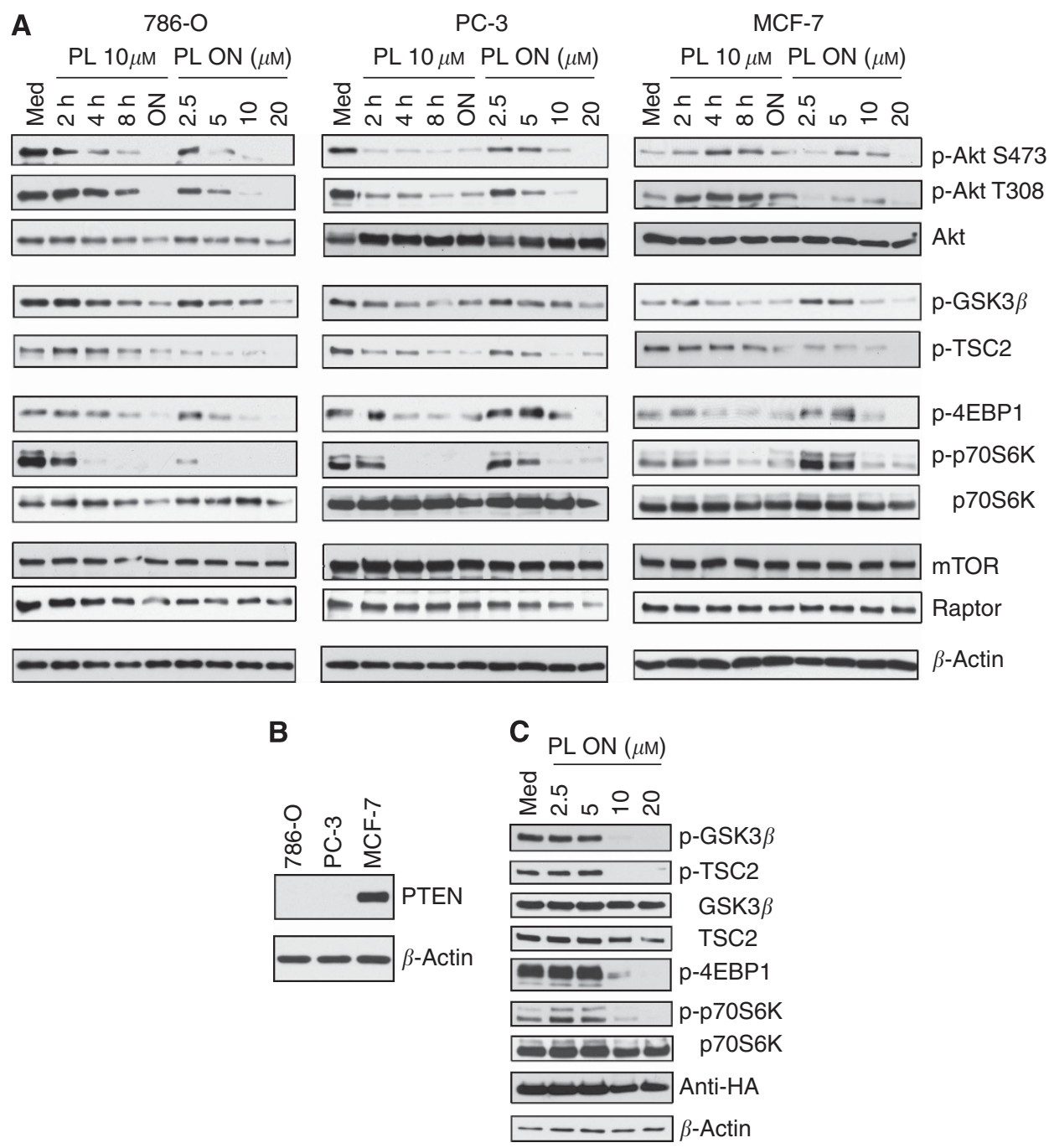

Figure 1. Piperlongumine affects Akt downstream signalling in cancer cells of various origins. (A) Piperlongumine decreases phosphorylation levels of Akt effectors, GSK-3 $\beta$ and TSC2. Additionally, PL treatment results in significant downregulation of mTORC1 complex activity but did not affect the main components of mTORC1 complex, mTOR and Raptor. Cells were treated with PL at indicated concentrations for 2, 4, $8 \mathrm{~h}$ or overnight (ON, 20-24 h). (B) Western blot analysis of PTEN expression in 786-O, PC-3 and MCF-7 cells. (C) The inhibitory effect of PL on functioning of constitutively active Akt(T308D; S473D) mutant in MCF-7 cells. MCF-7-HA-Akt ${ }^{\text {mut }}$ cells were treated with PL at indicated concentrations for $24 \mathrm{~h}$. Total cellular lysates were subjected to western blotting with the specific antibodies. 
$\left(100 \mu \mathrm{g} \mathrm{ml}^{-1}\right)$, sodium pyruvate $(1 \mathrm{~mm})$ and non-essential amino acids $(0.1 \mathrm{~mm})$ under conditions indicated in the figure legends.

Antibodies and reagents. Antibodies to Akt, phospho-Akt(S473), phospho-Akt(T308), phospho-Tuberin/TSC2(T1462), Tuberin/ TSC2, GSK-3 $\beta$, phospho-GSK-3 $\beta($ S9), mTOR, Raptor, phospho4E-BP1(T37/46), p70S6K, phospho-p70S6K(T389), LC3A/B, ULK1, phospho-ULK1(S757) and Anti-Rabbit IgG Fab2 Alexa Fluor (R) 488 conjugate were obtained from Cell Signaling Technology (Beverly, MA, USA). $\beta$-Actin and HA-probe antibodies were obtained from Santa Cruz Biotechnology Inc. (Santa Cruz, CA, USA). Piperlongumine was obtained from Indofine Chemical Company (Hillsborough, NJ, USA). Chloroquine diphosphate, Puromycin dihydrochloride, Hexadimethrine bromide and $\mathrm{N}$-Acetyl-L-Cysteine were obtained from Sigma-Aldrich (St Louis, MO, USA). Bafilomycine A1 was obtained from LC Laboratories (Woburn, MA, USA).

Western blot analysis. Whole-cell lysates preparation and western blot analysis were performed as described previously (Golovine et al, 2008).

Analysis of intracellular ROS production was performed as described previously (Golovine et al, 2013).

Establishment of PL ED50 for cancer cell lines. MCF-7 and 786-O cells were incubated with various PL concentrations for $48 \mathrm{~h}$. Cell proliferation was analysed by CellTiter Blue assay (Promega, Madison, WI, USA). Effective doses (ED) were calculated using XLift, Microsoft Excel add-in.
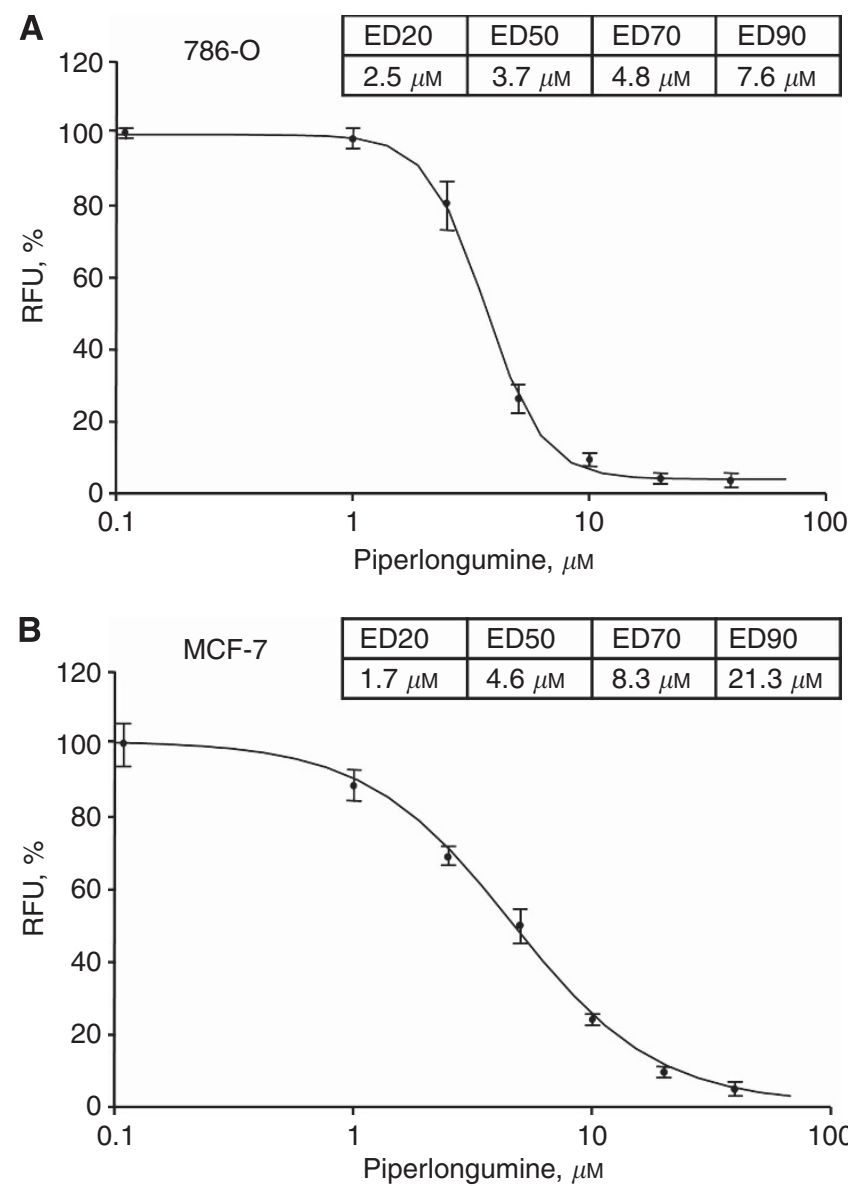

Figure 2. Piperlongumine demonstrates antiproliferative effect in 786-O (A) and MCF-7 (B) cells. Cells were treated with indicated concentrations of PL for $48 \mathrm{~h}$. Cellular proliferation was assessed using the CellTiter Blue assay.
Generation of MCF-7 cell line stably expressing constitutively active Akt. The mammalian expression vector pcDNA3 (plasmid 14751) encoding HA-tagged constitutively active Akt(T308D; S473D) mutant was obtained from Addgene (Cambridge, MA, USA). $\mathrm{HA}-\mathrm{Akt}^{\mathrm{mut}}$ ORF was then recloned into pLV-CMV-H4-puro lentiviral construct. HEK-293T cells were concomitantly transfected with plasmids, pLV-CMV-HA-Akt ${ }^{\text {mut }}$-H4-puro, pCMVdeltaR8.2 and pVSV-G (Clontech, Mountain View, CA, USA) using TransIT-293 Transfection reagent (Mirus, Madison, WI, USA). pLV-CMV-H4-puro and pCMVdeltaR8.2 constructs were kindly provided by Dr Alexey Ivanov (West Virginia University School of Medicine, Morgantown, WV, USA). Supernatant of HEK-293T cells containing lentiviral particles was collected $48 \mathrm{~h}$ after transfection, filtered through $0.22 \mu \mathrm{m}$ PVDF syringe filters and used for the infection of MCF-7 cells in presence of $4 \mu \mathrm{g} \mathrm{ml}^{-1}$ Polybrene (Hexadimethrine bromide). Forty-eight hours after infection, puromycin $\left(1 \mu \mathrm{g} \mathrm{ml}^{-1}\right)$ was added to the cellular media for further selection of infected cells.

Assessment of autophagy puncta formation by immunofluorescence. Immunofluorescent staining of LC3-II protein was carried out according to the protocol available at the official web site of Cell Signaling Technology (http://www.cellsignal.com/support/ protocols/IF_methanol.html). Confocal imaging was performed at room temperature using Nicon laser confocal microscope (model $\mathrm{C} 1$ ). Images were acquired using $\times 60$ oil lens using the EZ-C1 software package.

Analysis of cell death. The evaluation of cell death was performed using Propidium iodide (PI) staining with following Flow Cytometry analysis. Analysis was performed using FACScan (Becton Dickinson, Franklin Lakes, NJ, USA). Individual fluorescent populations were determined through the use of acquisition and analysis software (Cell Quest, Becton Dickinson).

Assessment of in vivo tumour growth. For in vivo studies, $1 \times 10^{6}$ PC-3 cells were inoculated s.c. in the flank region of 6-week-old male C.B17/Icr-scid mice using a 27-gauge needle. All animal procedures were done in accordance with the institutional guidelines on animal care and with appropriate institutional certification. Animals were fed an autoclaved AIN-93M diet (Harlan Teklad, Madison, WI, USA) and water ad libitum. Ten days after the injection of tumour cells, animals were randomly

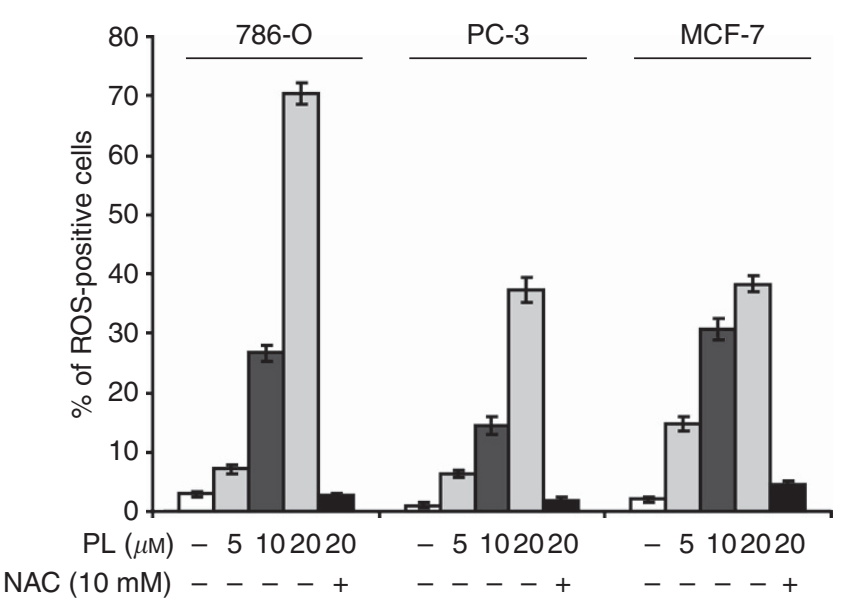

Figure 3. Treatment of 786-O, PC-3 and MCF-7 cells with PL results in increased intracellular ROS production, whereas addition of NAC completely blocks this process. Cells were treated with $\mathrm{PL}$ and/or NAC for $2 \mathrm{~h}$. Harvested cells were stained with $\mathrm{CM}-\mathrm{H}_{2}$ DCFDA and analysed by flow cytometry. The figure shows the representative data of one of three separate experiments (Bars, s.e.m. of triplicates). 
assigned to the control or experimental groups $(n=5$ mice per group). The mice were treated with (i) $0.9 \% \mathrm{NaCl}, 0.3 \%$ DMSO solution (vehicle); (ii) PL ( $20 \mathrm{mg} \mathrm{kg}^{-1}$ in $0.9 \% \mathrm{NaCl}, 0.3 \% \mathrm{DMSO}$ solution, daily, I.P.); (iii) CQ (40 $\mathrm{mg} \mathrm{kg}^{-1}$, in $0.9 \% \mathrm{NaCl}, 0.3 \%$ DMSO solution daily, I.P.); or (iv) with PL and CQ combined. Tumours were measured twice weekly and their volumes were calculated using the formula: (volume $=0.52 \times(\text { width })^{2} \times$ length).

\section{RESULTS}

Piperlongumine decreases the phosphorylation level of Akt and/or its downstream effectors. Recent publication reported the ability of PL to increase apoptotic cell death in cancer cells while exerting profound antitumour effects in vitro and in vivo (Raj et al, 2011). Taking into consideration that Akt promotes anti-apoptotic and pro-tumourigenic activities, we first turned our attention towards the investigation of the effects of PL on Akt downstream signalling. Three distinct organ cancer cell lines, prostate (PC-3), kidney (786-O) and breast (MCF-7) were chosen for the present study. The treatment of cells with PL resulted in a dramatic decrease of Akt/mTOR signalling. The phosphorylation levels of Akt downstream effectors, GSK-3 $\beta$ and TSC2, were notably depressed in all tested cell lines (Figure 1A). Moreover, PL-induced inhibition of Akt resulted in significant decrease of the mTORC1 complex activity, as made evident by the lowered phosphorylation levels of mTORC1 effectors, 4E-BP1 and p70S6K. Piperlongumine effects were uniformly time- and dose-dependent.

Notably, examination of the T308 and S473 phosphorylation levels of Akt in PL-treated cells yielded an alternate result. PC-3 and 786-O PL-treated cells exhibited decrease in phosphorylation levels both in time- and dose-dependent observations. Furthermore, PL-treated MCF-7 cells demonstrated a paradoxic increase T308 and S473 phosphorylation levels of Akt. This effect was reversed at concentration of $20 \mu \mathrm{M}$, suggesting that PL acts in a non-specific and toxic manner at those levels.
Regardless of the impact on phosphorylation of Akt, PL attenuated the phosphorylation of downstream Akt targets. Taken together, our data provide evidence that various downstream Akt effectors may be affected by PL administration, PL affects Akt phosphorylation in a cell-specific manner dependently on phosphatase and tensin homologue (PTEN) expression (Figure 1B); however, phosphorylation of Akt effectors was inhibited uniformly.

To further elucidate the effect of PL on Akt downstream targets and its impact on the upstream activation of Akt, we generated a stably expressing, HA-tagged, constitutively active Akt(T308D; S473D) mutant MCF-7 cell line (MCF-7-HA-Akt ${ }^{\text {mut }}$ ). MCF-7-HA-Akt ${ }^{\text {mut }}$ cells were treated with various concentrations of PL for a $24 \mathrm{~h}$ period. Predictably, PL treatment resulted in significant downregulation of GSK-3 $\beta$ and TSC2 phosphorylation levels. When administered at $10 \mu \mathrm{M}$ or higher, PL demonstrated an observable impact on downstream Akt effectors (Figure 1C). Additionally, PL-treated MCF-7-HA-Akt ${ }^{\text {mut }}$ cells showed a marked decrease in the mTORC1 activity level.

Cellular proliferation is affected by PL. 786-O and MCF-7 cell lines were administered with varying concentrations of PL to evaluate PL IC50. Relatively to PL mediated decrease of Akt effectors phosphorylation levels, PL affected cellular proliferation, dose-dependently (Figure 2). Similar results demonstrating that PL effectively blocks proliferation of PC-3 cells have been published recently (Ginzburg et al, 2013).

Elevation of ROS levels as a result of PL treatment is responsible for Akt inactivation. To examine whether PL affects the phosphorylation level of Akt effectors via the ROS pathway, we measured the level of intracellular ROS following treatment with PL. Cells were treated with increasing concentrations of PL alone or concomitantly with a well-established antioxidant, $\mathrm{N}$-Acetyl-L-Cysteine (NAC). Expectedly, the increase in ROS production in PL-treated cells was observed in a dose-dependent manner and was blocked by the addition of NAC to the cellular

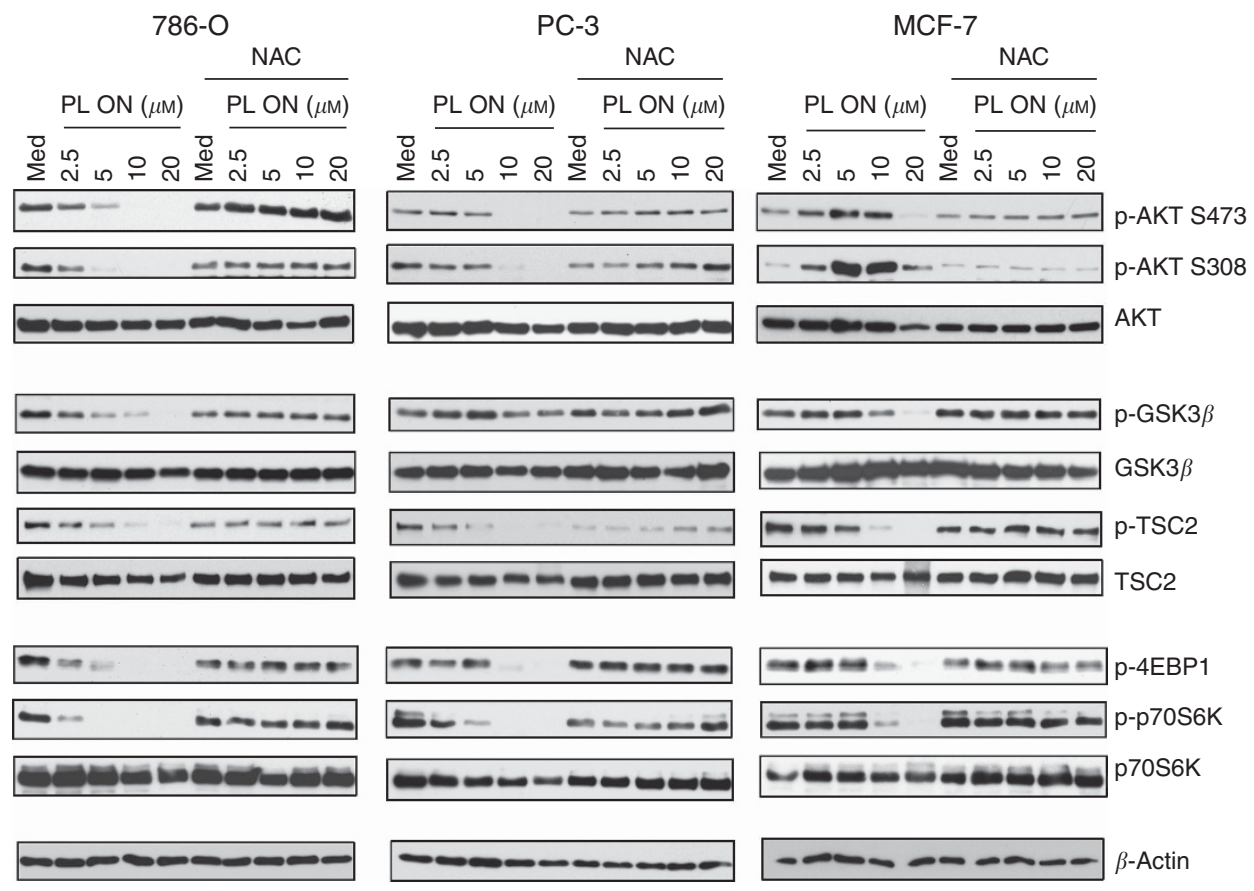

Figure 4. NAC reverses negative effects of PL on Akt downstream signalling. 786-O, PC-3 and MCF-7 cells were treated with PL at indicated concentrations alone or in combination with $10 \mathrm{~mm}$ of NAC for $24 \mathrm{~h}$. Total cellular lysates were subjected to western blotting with specific antibodies. 
medium (Figure 3). Furthermore, NAC administration completely reversed PL-induced Akt functional changes in every tested cell line. Figure 4 illustrates that PL-associated causes decrease in phosphorylation levels of Akt effectors, GSK-3 $\beta$ and TSC2, and mTORC1 target proteins, 4E-BP1 and p70S6K, were abolished in cells treated concomitantly with NAC. Treatment with NAC alone did not induce any changes in either phospho-GSK-3 $\beta$ and phospho-TSC2 protein levels, or in the phosphorylated forms of 4E-BP1 and p70S6K. Moreover, cells treated with excessive amounts of PL $(20 \mu \mathrm{M})$ were able to overcome its toxic effects following reversal with NAC. Our experimental data offer compelling evidence that PL exerts a strong negative effect on Akt downstream signalling through indirect stimulation of ROS.

Piperlongumine-induced inactivation of Akt/mTORC1 signalling promotes autophagy. After our initial data demonstrated the inhibitory effects of PL on the Akt/mTORC1 pathway, we extended our analysis to examine the role played by PL in the process of autophagy. Cells were treated with increased concentrations of PL alone or in presence of NAC (10 mM) for $24 \mathrm{~h}$. The microtubule-associated protein 1 light chain 3 (LC3) is widely used as a marker for autophagy (Tanida et al, 2004). Therefore, we performed western blot analysis utilising antiLC3A/B antibodies that preferentially bind LC3-II protein. We observed that LC3-II protein accumulation in all tested cells responded to the administration of PL (Figure 5A). Treatment with excessive amounts of PL $(20 \mu \mathrm{M})$ resulted in undetectable levels of LC3-II, further offering evidence of the deleterious/toxic effect of PL at high concentrations. Piperlongumine-mediated autophagy in all tested cell lines was ROS-dependent, made evident by the complete reversal of LC3-II accumulation (Figure 5A) and mTORC1 inhibition following the administration of NAC.

As ULK1, a mammalian autophagy-initiating kinase is directly controlled by mTORC1 (Kim et al, 2011), we additionally examined whether ULK1 serine 757 phosphorylation levels would be affected by PL treatment. Expectedly, PL treatment resulted in dramatic downregulation of the phospho-Ser757 ULK1 levels in all tested cell lines (Figure 5A).

Increased LC3-II levels may be observed during enhanced autophagosome formation or reduced autophagosome turnover (Rubinsztein et al, 2009). To further substantiate our results, we examined level changes of processed LC3-II in cells treated with PL alone and PL with a well-established autophagy inhibitor, Bafilomycine A1 (Baf-A1). Our data indicate that cells treated concomitantly with Baf-A1 and PL accumulate LC3-II at higher levels, further supporting PL's role as an autophagy inducer (Figure 5B). Data were further confirmed through the analysis of autophagosome formation by immunofluorescence using antiLC3A/B antibodies preferentially binding the type II form of LC3A/B. Providing further validation to our initial results, LC3 punctum formation was observed by fluorescent microscopy solely in cells treated with PL and temsirolimus (positive control) (Figure 6). Cells treated with concomitant PL and NAC showed a barely detectable LC3 punctum formation, mimicking untreated cells displaying basal autophagy levels.

Modulation of PL-mediated cell death by autophagy inhibitors. Autophagy promotes the survival of cells resistant to apoptosis when they are deprived of extracellular nutrients or growth factors and represents a mechanism of resistance to anticancer therapy-mediated cell death (Dikic et al, 2010). With that in mind, we turned our attention towards examining the effect of autophagy inhibition on PL-induced cell death. We utilised an established autophagy inhibitor, CQ, which has been shown to exhibit antitumour effects and sensitise cancer cells to chemotherapeutic drugs (Amaravadi et al, 2007; Firat et al,
A
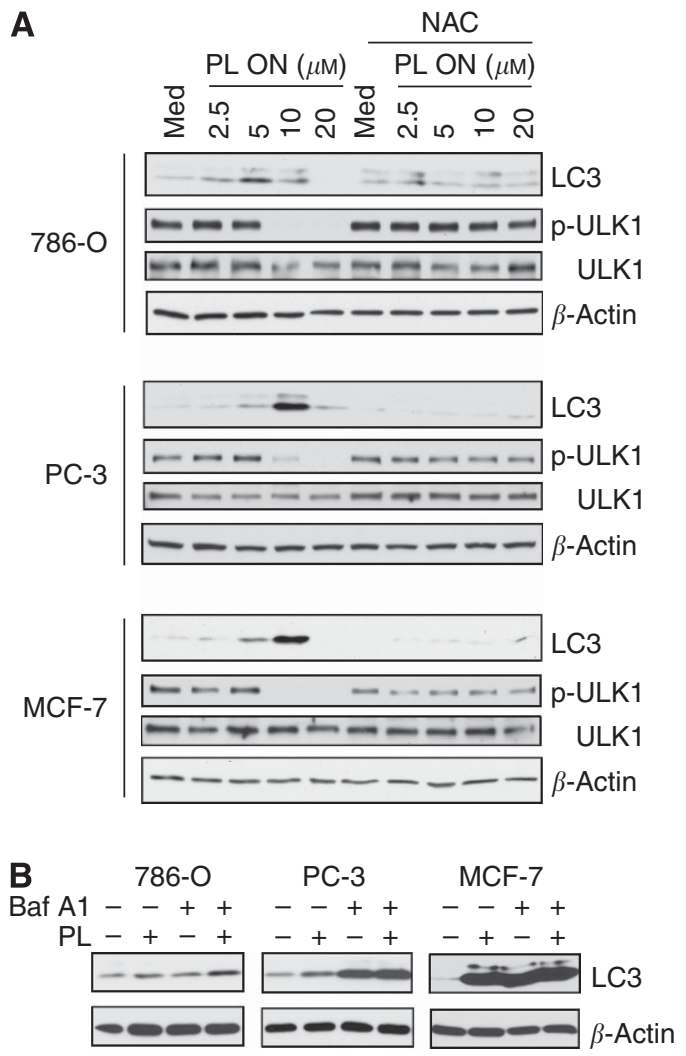

Figure 5. Treatment with PL promotes autophagy induction. (A) Piperlongumine treatment results in LC3-II accumulation and decrease of ULK1 Ser757 phosphorylation. Piperlongumine induction of autophagy is ROS dependent, as NAC completely reverses effects of PL. Cells were treated with indicated concentrations of $\mathrm{PL}$ alone or in combination with $10 \mathrm{~mm}$ of NAC for $24 \mathrm{~h}$. (B) Concomitant treatment of cells with PL $(10 \mu \mathrm{m})$ and Baf-A1 $(10 \mu \mathrm{M})$ results in higher accumulation of LC3-II then treatment with PL alone, which indicates PL acts as autophagy inducer rather than inhibitor. Total cellular lysates were subjected to western blotting with specific antibodies. Upper band (if visible) corresponds to LC3-I and lower band corresponds to LC3-II form of LC3 protein.

2012). Cells were treated with either $20 \mu \mathrm{M}$ of CQ alone, with $10 \mu \mathrm{M}$ of PL alone or concomitantly for $72 \mathrm{~h}$. Treatment with PL alone led to a measurable induction of cell death, whereas concomitant treatment with PL and CQ resulted in the most profound measured levels of cell death in all tested cell lines (Figure 7). These results bring forth a critical point that PLinduced cellular death can be enhanced through concurrent autophagy inhibition, CQ in this case.

Antitumour effects of PL enhanced by CQ in vivo. Our data presented above clearly demonstrate the ability of CQ to sensitise cancer cells to PL in vitro. We next, extended our findings by evaluation of antitumour effects of PL alone or in combination with CQ in vivo. Xenograft tumours were established in severe combined immunodeficient mice using PC-3 cells. Animals were administered CQ $\left(40 \mathrm{mg} \mathrm{kg}^{-1}\right)$ or PL $\left(20 \mathrm{mg} \mathrm{kg}^{-1}\right)$ daily through intraperitoneal injections as monotherapy, or as combination therapy at indicated doses. As demonstrated in Figure 8, treatment with CQ alone did not yield any significant tumour regression. Treatment with PL alone resulted in a statistically significant inhibition of tumour growth. Concomitant treatment with PL and $\mathrm{CQ}$, however, resulted in the most profound regression of tumour mass. 


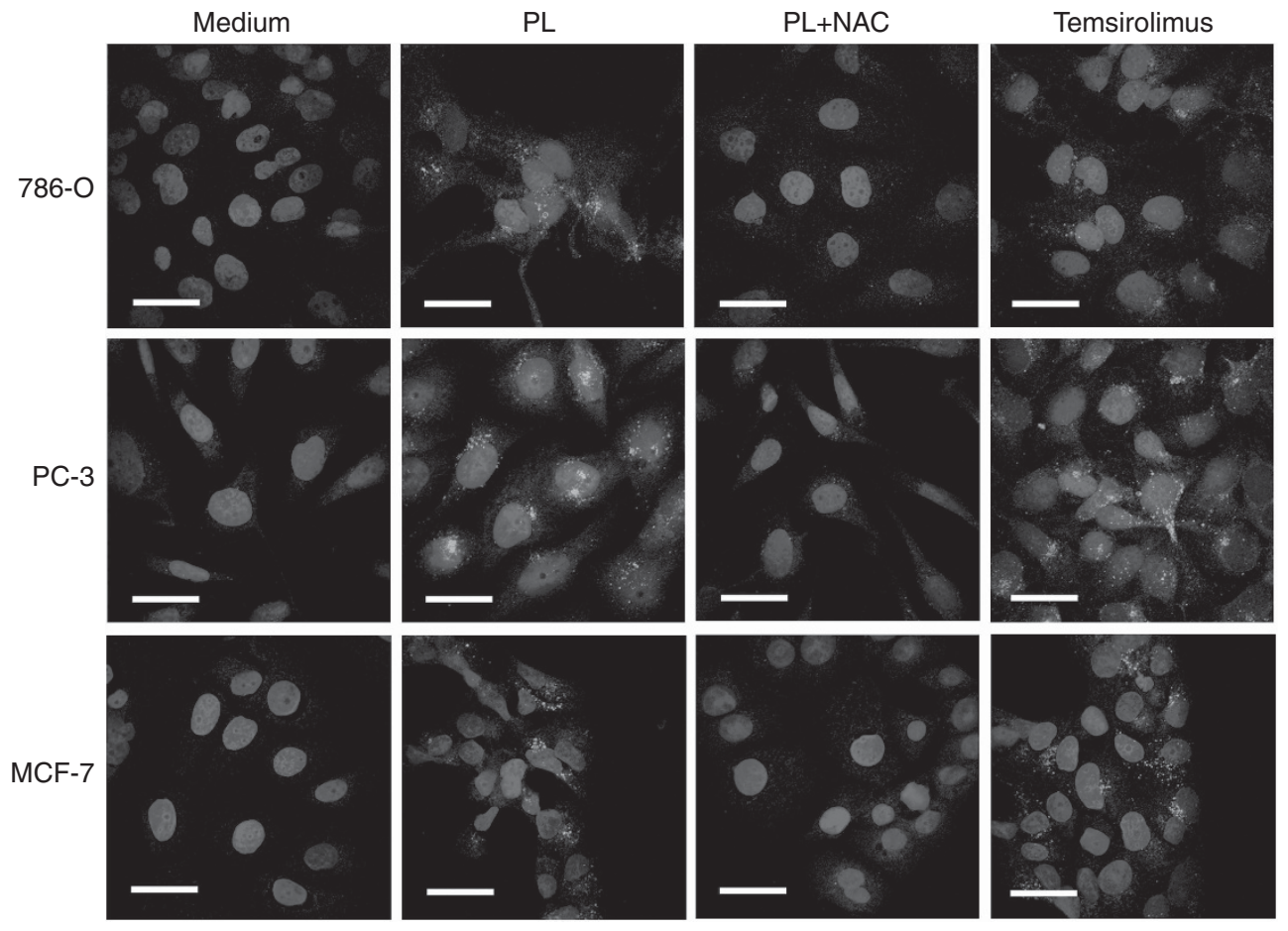

Figure 6. Immunofluorescent detection of increased autophagosome flux in cells treated with PL. N-Acetyl-L-Cysteine reverses the autophagyinducing effect of PL. Additionally, cells were treated with mTORC1 inhibitor, temsirolimus, which induced autophagy serving as a positive control. Light chain 3-II is shown in green and DAPI in blue. Bar, $50 \mu \mathrm{m}$. The full colour version of this figure is available at British Journal of Cancer online.
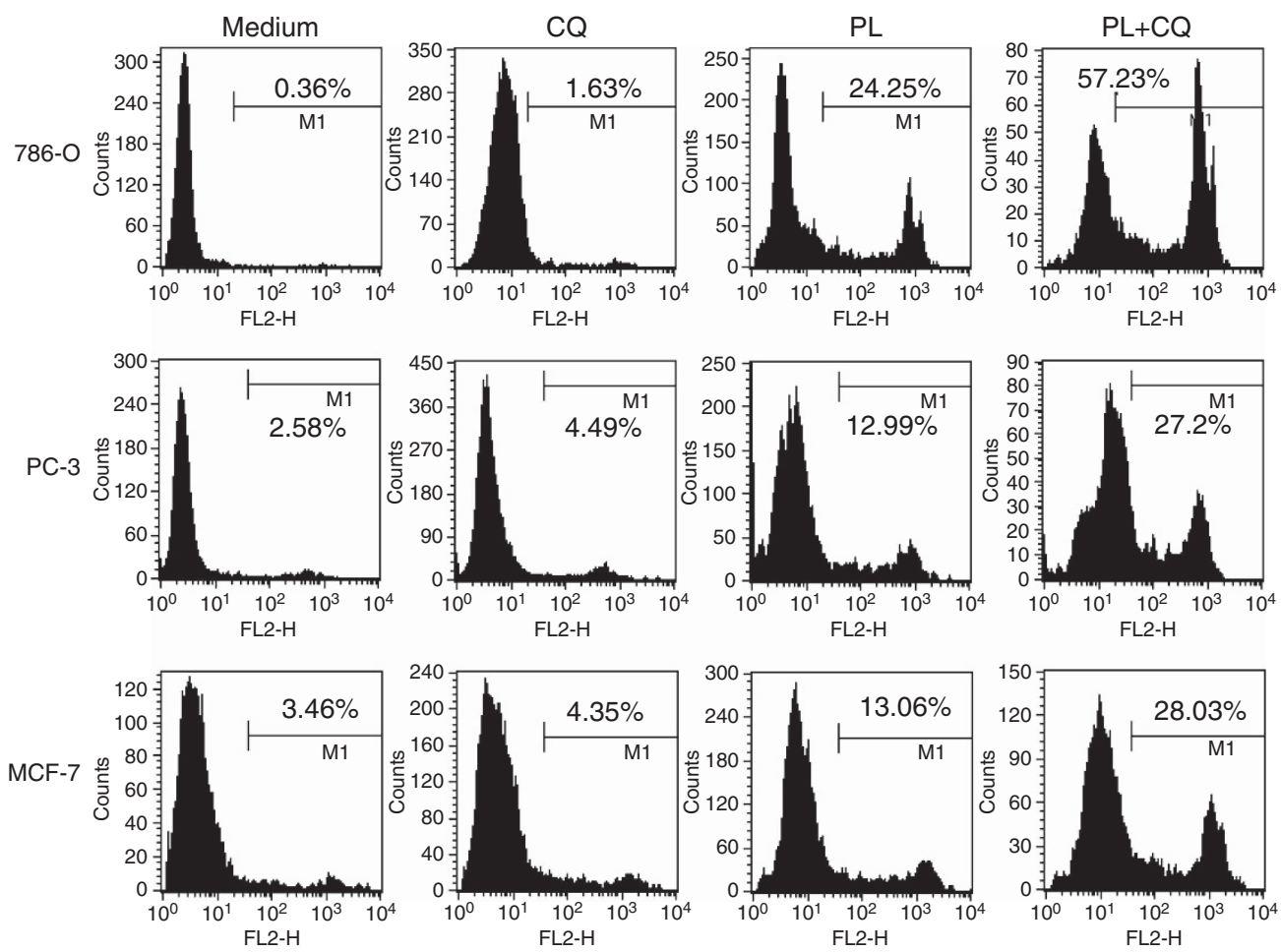

Figure 7. Inhibition of autophagy by CQ promotes PL-mediated cancer cell death in vitro. Cells were treated with either $20 \mu \mathrm{m}$ of $\mathrm{CQ}$ alone, with $10 \mu \mathrm{m}$ of PL alone or concomitantly for $72 \mathrm{~h}$. Cells were then harvested, PI was added to cellular suspensions at $3 \mu \mathrm{g} \mathrm{ml} \mathrm{C}^{-1} \mathrm{concentration}$ and analysed by Flow cytometry. The representative data from one of three independent experiments are presented. 


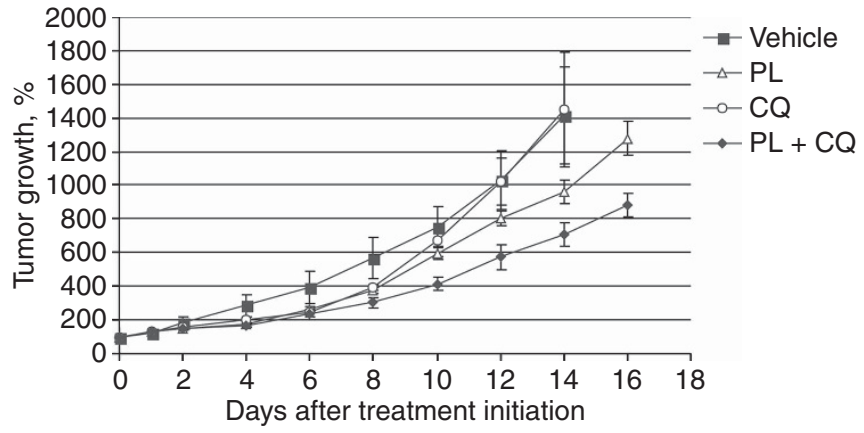

Figure 8. The concomitant treatment with $\mathrm{PL}$ and $\mathrm{CQ}$ results in inhibition of tumour growth xenograft mouse tumour model.

Subcutaneous PC-3 tumors were established in 6-week-old male C B17/Icr-scid mice. Treatment with PL and/or CQ and assessment of tumor growth were carried out as described in Materials and Methods. Data shown are mean of five mice in each group (s.e.m. displayed with bars).

\section{DISCUSSION}

Physiologically ROS are toxic by-products that are generated by the mitochondria via a multicomponent NADPH oxidase enzymatic complex of the respiratory chain (Balaban et al, 2005). To date, compelling evidence exists that points to ROS function as an important physiological regulator of intracellular signalling pathways (Ray et al, 2012).

Recent publications reveal the antitumour role of ROS, which is carried out through several distinct mechanisms. Reactive oxygen species has been linked to mediation of apoptosis through activation of JNK signalling (Whibley et al, 2007). In addition, recent work published by Raj et al (2011) demonstrates direct involvement of ROS in selective killing of cancer cells.

The Akt/mTOR signalling pathway has a crucial regulatory role in cellular proliferation and survival, glucose metabolism and angiogenesis (Manning and Cantley, 2007). A host of recent publications deal with the impact of ROS on Akt/mTOR signalling. Enhanced Akt signalling primarily via the ROS-mediated inactivation of PTEN has been well documented in multiple reports (Leslie, 2006; Yalcin et al, 2010; Shearn et al, 2011b). Other data elaborate that in addition to its positive modulating effect on Akt signalling, ROS is capable of exerting a direct target effect on Akt itself under conditions of oxidative stress (Murata et al, 2003; Hussain et al, 2011; Shearn et al, 2011a).

Our current work declares that PL-mediated ROS generation promotes an inhibitory response on Akt/mTOR signalling and is involved in autophagy induction. Indeed, we observed a dramatic effect on phosphorylation of Akt effectors across all tested cancer cell lines, following administration of PL. As an added validity to our hypothesis that PL inhibition of Akt/mTOR signalling is mediated by ROS, administration of a well-established antioxidant, NAC completely reversed all cytotoxic effects of PL.

In our results, we point out the diverse effects of PL on phosphorylation levels of S473 and T308 Akt sites. This is likely explained by cellular PTEN expression status and consistent with prior studies demonstrating inactivation of PTEN by ROS (Leslie, 2006). Furthermore, a strong possibility of positive feedback exists (Sun et al, 2005; O'Reilly et al, 2006), which explains the downregulation of mTORC1 activity in response to the inhibition of downstream Akt signalling. Indeed, when PTEN-positive MCF-7 cells were treated with PL, we observed enhanced Akt phosphorylation. Similar results were obtained when PTEN-positive cell lines of other origins, DU-145 (prostate cancer) and 769-P (kidney cancer) were treated with PL (data not presented). However, it would be hard to clarify whether PL has a direct inhibitory effect on mTOR kinase activity, or it may impair the mTORC1 complex integrity, or it may even affect other members of mTORC1 complex and dissect the data of mTORC1 itself functioning from dependence on Akt activity.

Phosphatase and tensin homologue-negative PC-3 and 786-O cells exhibit an independently high level of Akt phosphorylation even in the absence of strong upstream stimulation, which is necessary for overcoming the inhibitory role of PTEN (Ramaswamy et al, 1999). Interestingly, our results demonstrated the decrease of Akt S473 phosphorylation in PTEN-negative PC-3 and 786-O cells, indicating the potential possibility of PL ability to downregulate mTORC2 activity. However, it is difficult to clarify whether PL reduces the activity of mTORC2 complex, or PL reduces its upstream activation. Here we speculate that in PTENnegative cells, PL acts as a negative regulator of Akt; most likely through decrease in the upstream stimulation of mTORC2 complex rather than directly effecting mTORC2. This speculation is supported by the following observations: (a) PL treatment of PTEN-negative PC-3 and 786-O cells resulted in decrease in phosphorylation levels of both, p-Akt(T307) and p-Akt(S473); (b) PL did not show any negative effects on mTORC2 complex activity in PTEN-positive MCF-7 cells. Indeed, PTEN-negative cells are more sensitive to stimulation by growth factors (Sun et al, 1999) and upstream activation. Although PL-treated PTEN-positive MCF-7 cells demonstrate an associated rise in phospho-Akt levels, our data point out that Akt activity is in fact inhibited via a direct ROS-mediated effect. Piperlongumine-treated MCF-7 cells expressing constitutively active Akt revealed a similar decrease in phosphorylation level of Akt targets as in parental cells.

The role of the mTORC1 complex as a positive regulator of protein synthesis, cell growth and proliferation has been well established (Fingar and Blenis, 2004; Wang et al, 2005). Recent studies have shown that treatment with $\mathrm{H}_{2} \mathrm{O}_{2}$ and other oxidative agents increases the activation of Akt/mTOR signalling (Sarbassov and Sabatini, 2005; Li et al, 2010; Yoshida et al, 2011), whereas high doses of these agents have the reverse effect on mTORC1 activity (Kim et al, 2002; Liu et al, 2008; Li et al, 2010). Our data are in concert with the recently published data regarding negative redox regulation of mTORC1. Our group provides firsthand evidence that PL mediates mTORC1 inhibition via an Aktdependent mechanism.

Mammalian target of rapamycin complex 1 is also known for its ability to halt cellular catabolic processes such as autophagy (Kim et al, 2011). Expectedly, PL-mediated inhibition of mTORC1 resulted in autophagy induction in all tested cell lines, as demonstrated by the accumulation of LC3-II and decrease of serine 757 phosphorylation levels of ULK1 in PL-treated cells. In addition, our LC3-II accumulation assay was further reinforced by detection of autophagic puncta in cells treated with PL by immunofluorescence.

The link between autophagy and carcinogenesis has been well examined. Autophagy may provide cancer cells additional sources of energy and nutrients during periods of rapid tumour growth. Autophagy has been considered by some as type II programmed cell death, a concept which fell under heavy debate in recent years. The Nomenclature Committee of Cell Death 2009 points out that the term 'may misleadingly suggest a form of death occurring through autophagy, as this process often promotes cell survival' (Galluzzi et al, 2009). Presently recognised as a promoter of cancer cell survival, autophagy has become a target for the development of alternate anti-neoplastic strategies (Amaravadi et al, 2007; Apel et al, 2008; Palacios et al, 2010; Wu et al, 2010; Amaravadi et al, 2011). Our results further elaborate on the previously published data, providing clear evidence that inhibition of autophagy facilitates cancer cell death in response to anticancer therapy. Through our application of a well-established autophagy inhibitor, $\mathrm{CQ}$, we were able to significantly increase the level of cancer cell 
death when treated with PL. As added proof of principle, concomitant treatment with PL and CQ resulted in inhibition of xenograft model tumour growth.

Our work brings forth a number of key new concepts in an attempt to better understand the cellular alterations that take place following administration of PL. We provide first evidence that PL capably reduces the activity of Akt/mTOR signalling through generation of ROS, the effects of which are blocked by the administration of NAC. Furthermore, PL-induced Akt inactivation reduces mTORC1 activity subsequently promoting cell autophagy. In consistence with a number of recent data reporting cooperative inhibition of autophagy and PI3K/Akt/mTORC1 signalling pathway result in sensitization of cancer cells to the therapeutic stimuli (Degtyarev et al, 2008; Fan et al, 2010; Firat et al, 2012; Xie et al, 2013), our data provide strong support for the development of novel therapeutic strategies based on concomitant use of autophagy inhibitors and PL. This is a crucial concept, as it opens potential clinical avenues of modulating PL-induced cellular death through inhibition of autophagy.

\section{ACKNOWLEDGEMENTS}

We acknowledge the support of the Laboratory Animal, Biological imaging and Cell Sorting Facilities at Fox Chase Cancer Center. We thank Alexey Ivanov for the pLV-CMV-H4 puro, and pCMVdeltaR8.2 vectors. This work was supported in part by National Institutes of Health (Grant RO1 CA134463 and R03 CA167671 to VMK); FCCC/Temple Nodal Avard (to VMK); American Institute for Cancer Research Grant (to RGU); Department of Defence, Physician Research Training Award (W81XWH-10-1-0187 to AK) and Bucks County Board Associates Award (to AK).

\section{REFERENCES}

Alers S, Loffler AS, Paasch F, Dieterle AM, Keppeler H, Lauber K, Campbell DG, Fehrenbacher B, Schaller M, Wesselborg S, Stork B (2011) Atg13 and FIP200 act independently of Ulk1 and Ulk2 in autophagy induction. Autophagy 7(12): 1423-1433.

Amaravadi RK, Lippincott-Schwartz J, Yin XM, Weiss WA, Takebe N, Timmer W, DiPaola RS, Lotze MT, White E (2011) Principles and current strategies for targeting autophagy for cancer treatment. Clin Cancer Res 17(4): 654-666.

Amaravadi RK, Yu D, Lum JJ, Bui T, Christophorou MA, Evan GI, Thomas-Tikhonenko A, Thompson CB (2007) Autophagy inhibition enhances therapy-induced apoptosis in a Myc-induced model of lymphoma. J Clin Invest 117(2): 326-336.

Apel A, Herr I, Schwarz H, Rodemann HP, Mayer A (2008) Blocked autophagy sensitizes resistant carcinoma cells to radiation therapy. Cancer Res 68(5): 1485-1494.

Balaban RS, Nemoto S, Finkel T (2005) Mitochondria, oxidants, and aging Cell 120(4): 483-495.

Bezerra DP, Pessoa C, Moraes MO, Alencar NM, Mesquita RO, Lima MW, Alves AP, Pessoa OD, Chaves JH, Silveira ER, Costa-Lotufo LV (2008) In vivo growth inhibition of sarcoma 180 by piperlonguminine, an alkaloid amide from the Piper species. J Appl Toxicol 28(5): 599-607.

Debnath J, Baehrecke EH, Kroemer G (2005) Does autophagy contribute to cell death? Autophagy 1(2): 66-74.

Degtyarev M, De Maziere A, Orr C, Lin J, Lee BB, Tien JY, Prior WW, van Dijk S, Wu H, Gray DC, Davis DP, Stern HM, Murray LJ, Hoeflich KP, Klumperman J, Friedman LS, Lin K (2008) Akt inhibition promotes autophagy and sensitizes PTEN-null tumors to lysosomotropic agents. J Cell Biol 183(1): 101-116.

Dikic I, Johansen T, Kirkin V (2010) Selective autophagy in cancer development and therapy. Cancer Res 70(9): 3431-3434.

Fan QW, Cheng C, Hackett C, Feldman M, Houseman BT, Nicolaides T, Haas-Kogan D, James CD, Oakes SA, Debnath J, Shokat KM,
Weiss WA (2010) Akt and autophagy cooperate to promote survival of drug-resistant glioma. Sci Signal 3(147): ra81.

Fingar DC, Blenis J (2004) Target of rapamycin (TOR): an integrator of nutrient and growth factor signals and coordinator of cell growth and cell cycle progression. Oncogene 23(18): 3151-3171.

Firat E, Weyerbrock A, Gaedicke S, Grosu AL, Niedermann G (2012) Chloroquine or chloroquine-PI3K/Akt pathway inhibitor combinations strongly promote gamma-irradiation-induced cell death in primary stem-like glioma cells. PLoS One 7(10): e47357.

Galluzzi L, Aaronson SA, Abrams J, Alnemri ES, Andrews DW, Baehrecke EH, Bazan NG, Blagosklonny MV, Blomgren K, Borner C, Bredesen DE, Brenner C, Castedo M, Cidlowski JA, Ciechanover A, Cohen GM, De Laurenzi V, De Maria R, Deshmukh M, Dynlacht BD, El-Deiry WS, Flavell RA, Fulda S, Garrido C, Golstein P, Gougeon ML, Green DR, Gronemeyer H, Hajnoczky G, Hardwick JM, Hengartner MO, Ichijo H, Jaattela M, Kepp O, Kimchi A, Klionsky DJ, Knight RA, Kornbluth S, Kumar S, Levine B, Lipton SA, Lugli E, Madeo F, Malomi W, Marine JC, Martin SJ, Medema JP, Mehlen P, Melino G, Moll UM, Morselli E, Nagata S, Nicholson DW, Nicotera P, Nunez G, Oren M, Penninger J, Pervaiz S, Peter ME, Piacentini M, Prehn JH, Puthalakath H, Rabinovich GA, Rizzuto R, Rodrigues CM, Rubinsztein DC, Rudel T, Scorrano L, Simon HU, Steller H, Tschopp J, Tsujimoto Y, Vandenabeele P, Vitale I, Vousden KH, Youle RJ, Yuan J, Zhivotovsky B, Kroemer G (2009) Guidelines for the use and interpretation of assays for monitoring cell death in higher eukaryotes. Cell Death Differ 16(8): 1093-1107.

Ginzburg S, Golovine KV, Makhov PB, Uzzo RG, Kutikov A, Kolenko VM (2013) Piperlongumine inhibits NF-kappaB activity and attenuates aggressive growth characteristics of prostate cancer cells. Prostate 74(2): $177-186$.

Golovine K, Makhov P, Uzzo RG, Shaw T, Kunkle D, Kolenko VM (2008) Overexpression of the zinc uptake transporter hZIP1 inhibits nuclear factor-kappaB and reduces the malignant potential of prostate cancer cells in vitro and in vivo. Clin Cancer Res 14(17): 5376-5384.

Golovine KV, Makhov PB, Teper E, Kutikov A, Canter D, Uzzo RG, Kolenko VM (2013) Piperlongumine induces rapid depletion of the androgen receptor in human prostate cancer cells. Prostate 73(1): 23-30.

Gutierrez A, Snyder EL, Marino-Enriquez A, Zhang YX, Sioletic S, Kozakewich E, Grebliunaite R, Ou WB, Sicinska E, Raut CP, Demetri GD, Perez-Atayde AR, Wagner AJ, Fletcher JA, Fletcher CD, Look AT (2011) Aberrant AKT activation drives well-differentiated liposarcoma. Proc Natl Acad Sci USA 108(39): 16386-16391.

Han SS, Son DJ, Yun H, Kamberos NL, Janz S (2013) Piperlongumine inhibits proliferation and survival of Burkitt lymphoma in vitro. Leuk Res 37(2): $146-154$.

Hussain AR, Uddin S, Bu R, Khan OS, Ahmed SO, Ahmed M, Al-Kuraya KS (2011) Resveratrol suppresses constitutive activation of AKT via generation of ROS and induces apoptosis in diffuse large B cell lymphoma cell lines. PLoS One 6(9): e24703.

Kim DH, Sarbassov DD, Ali SM, King JE, Latek RR, Erdjument-Bromage H, Tempst P, Sabatini DM (2002) mTOR interacts with raptor to form a nutrient-sensitive complex that signals to the cell growth machinery. Cell 110(2): 163-175.

Kim J, Kundu M, Viollet B, Guan KL (2011) AMPK and mTOR regulate autophagy through direct phosphorylation of Ulk1. Nat Cell Biol 13(2): $132-141$.

Leslie NR (2006) The redox regulation of PI 3-kinase-dependent signaling. Antioxid Redox Signal 8(9-10): 1765-1774.

Levine B, Kroemer G (2008) Autophagy in the pathogenesis of disease. Cell 132(1): 27-42.

Li M, Zhao L, Liu J, Liu A, Jia C, Ma D, Jiang Y, Bai X (2010) Multi-mechanisms are involved in reactive oxygen species regulation of mTORC1 signaling. Cell Signal 22(10): 1469-1476.

Liu L, Wise DR, Diehl JA, Simon MC (2008) Hypoxic reactive oxygen species regulate the integrated stress response and cell survival. J Biol Chem 283(45): 31153-31162.

Majewski N, Nogueira V, Robey RB, Hay N (2004) Akt inhibits apoptosis downstream of BID cleavage via a glucose-dependent mechanism involving mitochondrial hexokinases. Mol Cell Biol 24(2): 730-740.

Manning BD, Cantley LC (2007) AKT/PKB signaling: navigating downstream. Cell 129(7): 1261-1274. 
Murata H, Ihara Y, Nakamura H, Yodoi J, Sumikawa K, Kondo T (2003) Glutaredoxin exerts an antiapoptotic effect by regulating the redox state of Akt. J Biol Chem 278(50): 50226-50233.

O'Reilly KE, Rojo F, She QB, Solit D, Mills GB, Smith D, Lane H, Hofmann F, Hicklin DJ, Ludwig DL, Baselga J, Rosen N (2006) mTOR inhibition induces upstream receptor tyrosine kinase signaling and activates Akt. Cancer Res 66(3): 1500-1508.

Opel D, Naumann I, Schneider M, Bertele D, Debatin KM, Fulda S (2011) Targeting aberrant PI3K/Akt activation by PI103 restores sensitivity to TRAIL-induced apoptosis in neuroblastoma. Clin Cancer Res 17(10): 3233-3247.

Palacios C, Martin-Perez R, Lopez-Perez AI, Pandiella A, Lopez-Rivas A (2010) Autophagy inhibition sensitizes multiple myeloma cells to 17-dimethylaminoethylamino-17-demethoxygeldanamycin-induced apoptosis. Leuk Res 34(11): 1533-1538.

Raj L, Ide T, Gurkar AU, Foley M, Schenone M, Li X, Tolliday NJ, Golub TR, Carr SA, Shamji AF, Stern AM, Mandinova A, Schreiber SL, Lee SW (2011) Selective killing of cancer cells by a small molecule targeting the stress response to ROS. Nature 475(7355): 231-234.

Ramaswamy S, Nakamura N, Vazquez F, Batt DB, Perera S, Roberts TM, Sellers WR (1999) Regulation of G1 progression by the PTEN tumor suppressor protein is linked to inhibition of the phosphatidylinositol 3-kinase/Akt pathway. Proc Natl Acad Sci USA 96(5): 2110-2115.

Ray PD, Huang BW, Tsuji Y (2012) Reactive oxygen species (ROS) homeostasis and redox regulation in cellular signaling. Cell Signal 24(5): 981-990.

Rubinsztein DC, Cuervo AM, Ravikumar B, Sarkar S, Korolchuk V, Kaushik S, Klionsky DJ (2009) In search of an "autophagomometer". Autophagy 5(5): 585-589.

Sarbassov DD, Sabatini DM (2005) Redox regulation of the nutrient-sensitive raptor-mTOR pathway and complex. J Biol Chem 280(47): 39505-39509.

Shearn CT, Fritz KS, Reigan P, Petersen DR (2011a) Modification of Akt2 by 4-hydroxynonenal inhibits insulin-dependent Akt signaling in HepG2 cells. Biochemistry 50(19): 3984-3996.

Shearn CT, Smathers RL, Stewart BJ, Fritz KS, Galligan JJ, Hail Jr. N, Petersen DR (2011b) Phosphatase and tensin homolog deleted on chromosome 10 (PTEN) inhibition by 4-hydroxynonenal leads to increased Akt activation in hepatocytes. Mol Pharmacol 79(6): 941-952.

Sun H, Lesche R, Li DM, Liliental J, Zhang H, Gao J, Gavrilova N, Mueller B, Liu X, Wu H (1999) PTEN modulates cell cycle progression and cell survival by regulating phosphatidylinositol 3,4,5,-trisphosphate and Akt/protein kinase B signaling pathway. Proc Natl Acad Sci USA 96(11): 6199-6204.
Sun SY, Rosenberg LM, Wang X, Zhou Z, Yue P, Fu H, Khuri FR (2005) Activation of Akt and eIF4E survival pathways by rapamycin-mediated mammalian target of rapamycin inhibition. Cancer Res 65(16): 7052-7058.

Tanida I, Ueno T, Kominami E (2004) Human light chain 3/MAP1LC3B is cleaved at its carboxyl-terminal Met121 to expose Gly120 for lipidation and targeting to autophagosomal membranes. J Biol Chem 279(46): 47704-47710.

Wang X, Beugnet A, Murakami M, Yamanaka S, Proud CG (2005) Distinct signaling events downstream of mTOR cooperate to mediate the effects of amino acids and insulin on initiation factor 4E-binding proteins. Mol Cell Biol 25(7): 2558-2572.

Whibley CE, McPhail KL, Keyzers RA, Maritz MF, Leaner VD, Birrer MJ, Davies-Coleman MT, Hendricks DT (2007) Reactive oxygen species mediated apoptosis of esophageal cancer cells induced by marine triprenyl toluquinones and toluhydroquinones. Mol Cancer Ther 6(9): 2535-2543.

White E, DiPaola RS (2009) The double-edged sword of autophagy modulation in cancer. Clin Cancer Res 15(17): 5308-5316.

Wu Z, Chang PC, Yang JC, Chu CY, Wang LY, Chen NT, Ma AH, Desai SJ, Lo SH, Evans CP, Lam KS, Kung HJ (2010) Autophagy blockade sensitizes prostate cancer cells towards src family kinase inhibitors. Genes Cancer 1(1): 40-49.

Xie X, White EP, Mehnert JM (2013) Coordinate autophagy and mTOR pathway inhibition enhances cell death in melanoma. PLoS One 8(1): e55096.

Yalcin S, Marinkovic D, Mungamuri SK, Zhang X, Tong W, Sellers R, Ghaffari S (2010) ROS-mediated amplification of AKT/mTOR signalling pathway leads to myeloproliferative syndrome in Foxo3(-/-) mice. EMBO J 29(24): 4118-4131.

Yang YC, Lee SG, Lee HK, Kim MK, Lee SH, Lee HS (2002) A piperidine amide extracted from Piper longum $L$. fruit shows activity against Aedes aegypti mosquito larvae. J Agric Food Chem 50(13): 3765-3767.

Yoshida S, Hong S, Suzuki T, Nada S, Mannan AM, Wang J, Okada M, Guan KL, Inoki K (2011) Redox regulates mammalian target of rapamycin complex 1 (mTORC1) activity by modulating the TSC1/TSC2-Rheb GTPase pathway. J Biol Chem 286(37): 32651-32660.

This work is published under the standard license to publish agreement. After 12 months the work will become freely available and the license terms will switch to a Creative Commons AttributionNonCommercial-Share Alike 3.0 Unported License. 